\title{
Resolvin D1 Reduces Emphysema and Chronic Inflammation
}

\author{
Hsi-Min Hsiao, ${ }^{*}$ Thomas H. Thatcher, ${ }^{\dagger \dagger}$ Romain A. Colas, ${ }^{\S}$ Charles N. Serhan, ${ }^{\S}$ Richard P. Phipps, ${ }^{\dagger \uparrow}$ and Patricia J. Sime ${ }^{\dagger \dagger \pi}$

\begin{abstract}
From the Departments of Pathology and Laboratory Medicine* and Environmental Medicine, ${ }^{\top}$ the Lung Biology and Disease Program, ${ }^{\dagger}$ and the Division of Pulmonary and Critical Care Medicine, ${ }^{\ddagger}$ University of Rochester School of Medicine and Dentistry, Rochester, New York; and the Center for Experimental Therapeutics and Reperfusion Injury, ${ }^{\S}$ Department of Anesthesiology, Perioperative, and Pain Medicine, Brigham and Women's Hospital, Harvard Institutes
\end{abstract} \\ of Medicine and Harvard Medical School, Boston, Massachusetts
}

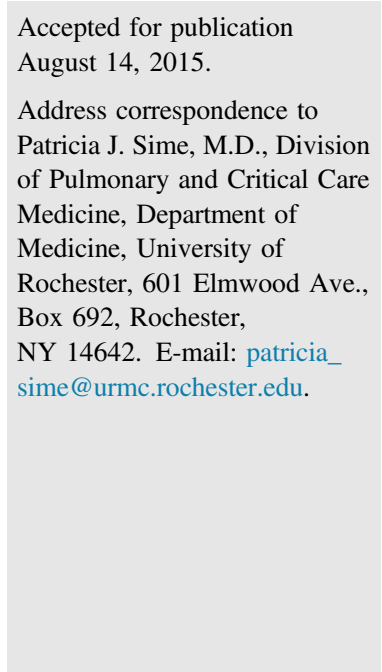

Accepted for publication August 14, 2015.

Address correspondence to Patricia J. Sime, M.D., Division of Pulmonary and Critical Care Medicine, Department of Medicine, University of Rochester, 601 Elmwood Ave., Box 692, Rochester, NY 14642. E-mail: patricia sime@urmc.rochester.edu.

\begin{abstract}
Chronic obstructive pulmonary disease is characterized, in part, by chronic inflammation that persists even after smoking cessation, suggesting that a failure to resolve inflammation plays an important role in the pathogenesis of the disease. It is widely recognized that the resolution of inflammation is an active process, governed by specialized proresolving lipid mediators, including lipoxins, resolvins, maresins, and protectins. Here, we report that proresolving signaling and metabolic pathways are disrupted in lung tissue from patients with chronic obstructive pulmonary disease, suggesting that supplementation with proresolving lipid mediators might reduce the development of emphysema by controlling chronic inflammation. Groups of mice were exposed long-term to cigarette smoke and treated with the proresolving mediator resolvin D1. Resolvin D1 was associated with a reduced development of cigarette smoke-induced emphysema and airspace enlargement, with concurrent reductions in inflammation, oxidative stress, and cell death. Interestingly, resolvin D1 did not promote the differentiation of M2 macrophages and did not promote tissue fibrosis. Taken together, our results suggest that cigarette smoking disrupts endogenous proresolving pathways and that supplementation with specialized proresolving lipid mediators is an important therapeutic strategy in chronic lung disease, especially if endogenous specialized proresolving lipid mediator signaling is impaired. (Am J Pathol 2015, 185: 3189-3201; http:// dx.doi.org/10.1016/j.ajpath.2015.08.008)
\end{abstract}

Chronic obstructive pulmonary disease (COPD) is a major global health problem and a leading cause of death and disability. Tobacco smoking remains the major causative factor in the development of COPD; however, new evidence suggests that household air pollution from fuel used for indoor cooking and heating is also a significant cause. ${ }^{1,2}$ COPD includes chronic bronchitis (with a clinical presentation of airway constriction), small airway remodeling, and emphysema, a pathological outcome of parenchymal destruction due to unresolved chronic inflammation. Despite the deadly nature of COPD, treatment options are scarce. Current treatments, ranging from corticosteroids and bronchodilators to lung volume reduction surgery, are only able to alleviate symptoms. There are no effective treatments that reverse or even slow the progression of the ultimately fatal course of the disease; therefore, new treatment options are desperately needed. ${ }^{3}$ Interestingly, once chronic bronchitis and emphysema have developed, the disease can progress even after the patient stops smoking. ${ }^{4,5}$ Therefore, it is important to investigate the underlying resolution circuits so that we can have a better understanding of the pathology of COPD and can devise new and effective treatment strategies.

Supported by NIH grants R01HL120908, T32HL066988, P30ES001247, and GM038765 (C.N.S.). The project described was supported by National Center for Advancing Translational Sciences of the National Institutes of Health award UL1TR000042 (H.H.).

The content is solely the responsibility of the authors and does not necessarily represent the official views of the National Center for Research Resources or the NIH.

Disclosures: C.N.S. is an inventor on patents (resolvins) assigned to Brigham and Women's Hospital (Boston, MA) and licensed to Resolvyx Pharmaceuticals (Cambridge, MA). C.N.S. is a scientific founder of Resolvyx Pharmaceuticals and owns equity in the company. The interests of C.N.S. were reviewed and are managed by the Brigham and Women's Hospital and Partners HealthCare in accordance with their conflicts-of-interest policies. 
Chronic inflammation is implicated as a key pathological factor in COPD, contributing to airway obstruction, parenchymal destruction (emphysema), and activation of proteases, oxidative and nitrosative stress, apoptosis, and altered macrophage properties. ${ }^{4,5}$ COPD patients exhibit multiple indications of chronic inflammation, including elevated lung macrophages, neutrophils, $\mathrm{T}$ cells, and proinflammatory mediators including IL-8 [chemokine (C-X-C motif) ligand 8; CXCL8], monocyte chemotactic protein 1 (MCP-1/CCL2), and IL-6. ${ }^{5,6}$ Oxidative stress is also an important pathogenic factor in COPD, as reactive oxygen and nitrogen species have long been known to cause lipid peroxidation, protein oxidation, and DNA mutation, eventually leading to cell cycle arrest and cell death. ${ }^{4}$ Although cigarette smoke contains numerous short-acting reactive oxygen species and reactive nitrogen species, it is underappreciated that inflammatory cells themselves are a major contributor to tissue oxidative stress in chronic inflammation. Activated macrophages and neutrophils can produce superoxide, nitric oxide, peroxynitrite, hydrogen peroxide, and hypochlorite..$^{5,7,8}$ This production generates the potential for a proinflammatory feed-forward loop that promotes continued chronic inflammation and tissue damage even after smoking cessation. ${ }^{4}$

It is now widely acknowledged that the resolution of inflammation is a programed, self-limited process governed by a group of bioactive lipid mediators, coined specialized proresolving lipid mediators (SPMs). ${ }^{9}$ SPMs are mainly biosynthesized from $\omega-3$ essential fatty acids, including eicosapentaenoic and docosahexaenoic acids, and are generally categorized into five families: lipoxins, D-series resolvins, E-series resolvins, protectins, and maresins. ${ }^{9}$ It has been observed that a diet enriched in $\omega-3$ essential fatty acids improved lung function and reduced symptoms in smokers who had COPD, suggesting potential beneficial roles of SPMs in the lung. ${ }^{10}$ Resolvin D1 (RvD1) is a D-series SPM so named as it is biosynthesized from docosahexaenoic acid. Previously, we reported that RvD1 inhibits acute lung inflammation elicited by cigarette smoke exposure and facilitates the resolution of inflammation after smoke exposure by limiting inflammatory cell infiltration and promoting macrophage phagocytosis. ${ }^{11}$ Others have shown that RvD1 attenuates LPS-induced and acid-induced acute lung injury, as well as allergic airway inflammation. ${ }^{11-13}$ These results suggest that RvD1 plays a role in resolution and homeostasis in the lung and that RvD1 might be used for treating inflammatory lung disease caused by harmful inhaled toxicants and microorganisms.

Although the role of RvD1 in acute inflammatory settings has been investigated, whether RvD1 signaling plays a role in chronic inflammatory diseases such as COPD remains largely unknown. Here, we investigated whether RvD1 signaling is involved in the pathogenesis of COPD and whether exogenous RvD1 reduces chronic inflammation and emphysema in a mouse model of long-term cigarette smoke exposure.

\section{Materials and Methods}

\section{Reagents and Antibodies}

Aspirin-triggered (AT)-RvD1 (17R-RvD1; 7S, 8R, 17Rtrihydroxy-4Z, 9E, 11E, 13Z, 15E19Z-docosahexaenoic acid) was purchased from Cayman Chemical (Ann Arbor, MI). Rabbit anti-human lipoxin $\mathrm{A}_{4}$ receptor/formyl peptide receptor 2 (Alx/Fpr2) (ab63022), $\beta$-tubulin (ab151318), nitrotyrosine (ab78163), and anti-8-hydroxydeoxyguanosine (8-OHdG; Ab10802) were purchased from Abcam (Cambridge, MA). Rabbit anti-mouse Alx/Fpr2 (sc-66901) was purchased from Santa Cruz Biotechnology (Santa Cruz, CA). Phycoerythrinconjugated anti-mouse Gr-1 (553128), biotinylated rat antimouse CD45 (553078), and CD16/CD32 (FcrIII/II receptor; 553142) were purchased from BD Pharmingen (San Jose, CA). Alexa Fluor 488 and 647-conjugated anti-mouse F4/80 (MF48020 and MF48021, respectively) and Alexa Fluor 568-conjugated streptavidin were purchased from Life Technologies (Grand Island, NY). Anti-eicosanoid oxidoreductase (Eor; NB200-179) was purchased from Novus Biologicals (Littleton, CO). Anti-mouse cyclooxygenase-2 (Cox-2) antibody (160126) was purchased from Cayman Chemical. Rabbit polyclonal antibody Ho-1 (SPA-896) was purchased from Enzo Life Sciences (Farmingdale, NY).

\section{Human Tissue Samples}

Human lung tissue samples were collected from patients with or without COPD who were undergoing diagnostic biopsy for suspected lung mass. Tissue as distal to the lesion as possible was used. Donors were consented based on the availability of the surgeon and the availability of our clinical coordinator to obtain consent and process the samples. All samples collected for this study (four non-COPD and five COPD) are reported here. Human tissue collection was performed under the supervision of the University of Rochester Research Subjects Review Board, and all donors gave informed written consent. The donor characteristics are summarized in Table 1.

\section{Lipid Mediator Profiling}

C57BL/6 mice (groups of three) were exposed to dilute mainstream cigarette smoke for 2 hours/day for 3 days, as previously described. ${ }^{11}$ The mice were euthanized with Avertin as described in Morphological and Morphometric Analysis. Left lungs were each placed in cold methanol and extracted as described followed by targeted lipid mediator-SPM profiling using liquid chromatography with tandem mass spectrometry. ${ }^{14,15}$

\section{Western Blot Analysis}

Collected human lung tissues were homogenized in sterile $\mathrm{H}_{2} \mathrm{O}$ supplemented with protease inhibitors (P8340; SigmaAldrich, St Louis, MO) and a phosphatase inhibitor (P0044; 
Table 1 Characteristics of Lung Tissue Donors

\begin{tabular}{|c|c|c|c|c|c|c|c|c|}
\hline Donor type & Age & Sex & Smoking & $\begin{array}{l}\text { Years since } \\
\text { quitting }\end{array}$ & Diagnosis & $\begin{array}{l}\text { DLC0, \% } \\
\text { predicted }\end{array}$ & $\begin{array}{l}\mathrm{FEV}_{1}, \% \\
\text { predicted }\end{array}$ & $\begin{array}{l}\text { FVC, \% } \\
\text { predicted }\end{array}$ \\
\hline \multicolumn{9}{|l|}{ Non-COPD } \\
\hline $\mathrm{N} 1$ & 45 & $\mathrm{~F}$ & Never & & Metastatic melanoma & 79 & 86 & 91 \\
\hline N2 & 28 & M & Current (14 py) & & Metastatic osteosarcoma & 72 & 110 & 118 \\
\hline N3 & 79 & M & Ex (64 py) & 18 & Metastatic colon cancer & ND & 86 & 91 \\
\hline N4 & 63 & $\mathrm{~F}$ & Ex & 25 & Non-small cell lung cancer & ND & 78 & 84 \\
\hline $\mathrm{C} 2$ & 72 & $\mathrm{~F}$ & Ex (35 py) & 4 & Adenocarcinoma & 29 & 89 & ND \\
\hline $\mathrm{C} 3$ & 80 & M & Ex (40 py) & 18 & No malignancy & 29 & 44 & 65 \\
\hline C4 & 54 & M & Current (29 py) & & Benign hamartoma & 74 & ND & 82 \\
\hline $\mathrm{C} 5$ & 60 & $\mathrm{~F}$ & Ex (25 py) & 8 & Adenocarcinoma & 52 & 78 & ND \\
\hline
\end{tabular}

Years since quitting were not recorded in all patients.

$\mathrm{F}$, female; $\mathrm{M}$, male; COPD, chronic obstructive pulmonary disease; DLCO, diffusing capacity of the lungs for carbon monoxide; $\mathrm{FEV}_{1}$, forced expiratory volume in 1 second; FVC, forced vital capacity; ND, not determined or not recorded; py, pack-years.

Sigma-Aldrich). Protein concentration was quantitated using a bicinchoninic acid detection assay (Pierce, Rockford, IL). Western blot analysis was performed as described previously. $^{11}$

\section{Exposure to Cigarette Smoke and RvD1 Treatment Regimen}

All animal experiments were performed under the supervision of the University of Rochester University Committee on Animal Research. Groups of mice were exposed to dilute mainstream cigarette smoke for 5 hours per day, 5 days per week, for 12 weeks. Smoke was generated using 3R4F reference cigarettes (University of Kentucky Reference Cigarette Program) smoked according to the Federal Trade Commission protocol (1 puff per minute of 35-mL volume per cigarette) and diluted with filtered room air using a Baumgartner-Jaeger CSM2072i automated smoking machine $(\mathrm{CH}$ Technologies, Westwood, NJ) as previously described. ${ }^{11,16}$ The number of cigarettes loaded in the carousel and the flow rate of dilution air were adjusted to give a nominal smoke exposure of $250 \mathrm{mg} / \mathrm{m}^{3}$ total particulate matter; the mean concentration of these exposures was $265 \pm 48 \mathrm{mg} / \mathrm{m}^{3}$. Control animals were exposed to filtered room air.

For treatment with AT-RvD1, air- and smoke-exposed mice were assigned randomly to receive AT-RvD1 by inhalation or i.v. injection, or vehicle only ( 80 mice total, 10 mice per group). In our previous study, ${ }^{11}$ we determined that $100 \mathrm{ng}$ of ATRvD1 given daily over 4 days was well tolerated and effective in inhibiting acute cigarette smoke-induced inflammation. We also conducted a pilot study (data not shown) that compared AT-RvD1 $100 \mathrm{ng}$ daily to a single 500-ng dose at the beginning of a 4-day acute-inflammation protocol and determined that the single dose was effective for at least 4 days, with no discernable adverse effects. Therefore, in this experiment we delivered AT-RvD1 500 ng twice per week. AT-RvD1 was received as a $100 \mu \mathrm{g} / \mathrm{mL}$ solution in $100 \%$ ethanol. For inhalation, AT-RvD1
$500 \mathrm{ng}$ was diluted in $40 \mu \mathrm{L}$ of normal saline (final ethanol concentration, $12.5 \%$ ) and delivered by oropharyngeal aspiration under isoflurane anesthesia. ${ }^{17}$ Control (vehicle) mice received $40 \mu \mathrm{L}$ of $12.5 \%$ ethanol in saline. For i.v. injection, AT-RvD1 $1000 \mathrm{ng}$ was diluted in $100 \mu \mathrm{L}$ of saline (final ethanol concentration, 10\%) and injected via the tail vein. Control (vehicle) mice received $100 \mu \mathrm{L}$ of $10 \%$ ethanol in saline. All groups of mice were treated with AT-RvD1 or vehicle twice per week for 12 weeks.

\section{Morphological and Morphometric Analysis}

After exposure to cigarette smoke for 12 weeks, mice were anesthetized with an i.p. injection of Avertin (2,2,2tribromoethanol, $250 \mathrm{mg} / \mathrm{kg}$ ), followed by exsanguination and removal of the lungs for analysis. The right bronchus was tied off, the right lobe was removed and used for other analyses, and the left lobe was inflated with $0.5 \%$ lowmelting agarose at $25 \mathrm{~cm}$ pressure, then fixed in $10 \%$ buffered formalin overnight. The left lobe was trimmed randomly into four pieces. Sections $(5 \mu \mathrm{m})$ from each cut face were stained with hematoxylin and eosin and imaged using a systematic random incremental sampling method. ${ }^{18}$ For measurement of mean linear intercept, 10 to 15 images per lung were acquired with an Olympus BM-51 microscope (Olympus Corporation, Center Valley, PA) fitted with a Spot Insight Camera (Spot Imaging Solutions, Sterling Heights, MI), using a $20 \times$ objective lens. A calibrated grid was laid over the images, the number of times the grid intercepted an alveolar wall was counted by two observers (H.H. and a technician who is not an author) who were blinded to the sample identities, and the mean linear intercept was determined as previously described. ${ }^{18}$ For calculation of the ratio of surface area to lung volume, mean cord length, total alveolar area, and septal length, a minimum of 15 images per lung were acquired using a Zeiss microscope $(10 \times$ objective lens) fitted with a AxioCam HRc camera (Zeiss, Oberkochen, Germany) by an investigator who was 
blinded to the sample identities (H.H.). The images were processed using MetaMorph Microscopy Automation and Image Analysis software version 7.8.8 (Molecular Devices, Sunnyvale, CA), with an algorithm generously gifted by Drs. Tiru Rangasamy and Rubin Tuder. ${ }^{19}$

\section{Lung Macrophage Isolation}

Four or five right lungs from each group were digested in Hanks balanced salt solution containing $1 \mathrm{mg} / \mathrm{mL}$ collagenase (SigmaAldrich) and $25 \mathrm{U} / \mathrm{mL}$ of DNase I (Sigma-Aldrich). A singlecell suspension was then prepared. Cells were first incubated with CD16/32 antibody to block the Fc receptor-mediated and nonspecific interactions. Cells were then washed and stained with fluorescein isothiocyanate-conjugated $\mathrm{F} 4 / 80$ and phycoerythrin-conjugated Gr-1. A FACSCanto II flow cytometer (BD Biosciences) was used for flow cytometric characterization of cell populations. Forward and side scatter was used for excluding doublets and cell debris. Mouse lung macrophages were isolated using cell-sorting technology from the whole-mouse lung digest using an Alexa 647-conjugated F4/80 antibody. Cell sorting was performed with an FACSVantage SE flow cytometer equipped with a DiVA sort option and an argon-ion laser at $488 \mathrm{~nm}$ excitation wavelength with a laser output of $200 \mathrm{~mW}$ (BD Biosciences). The BD FACSDiVa software package version 6.1.3 was used for data analysis (BD Biosciences). Purity of sorted cells was $\geq 98 \%$, as determined by flow cytometry. Total RNA was isolated from sorted macrophages using the RNeasy kit (Qiagen, Valencia, CA) according to the manufacturer's instructions.

\section{Total Lung RNA Isolation and Western Blot Analysis}

Four or five right lobes per group were cut into pieces and used for preparing total protein lysates for Western blot analysis and total lung RNA for quantitative PCR as described previously. ${ }^{11}$ Primer sequences of the genes we investigated in the study were: Il6, 5'-GGCGGATCGGATGTTGTGAT-3' (forward), 5'-GGACCCCAGACAATCGGTTG-3' (reverse); Cxcll (keratinocyte chemoattractant), 5'-ACTGCACCCAAACCGAAGTC-3' (forward), 5'-TGGGGACACCTTTTAGCATCTT-3' (reverse); $C c l 2$ (monocyte chemotactic protein 1), 5'-TAAAAACCTGGATCGGAACCAAA-3' (forward), $5^{\prime}$-GCATTAGCTTCAGATTTACGGGT- $3^{\prime}$ (reverse); and Alox15, 5'-TCCCTCAACCTAGTGCGTTTG-3' (forward), 5'-CCTCGGGAACGTCGAAGTC-3' (reverse). Primer sequences for Argl, Mrcl, Tnfa, iNos (Nos2) and 18sRNA were described previously. ${ }^{11}$

\section{TUNEL Assay}

Apoptotic cells were detected by the DeadEnd Colorimetric TUNEL System (Promega, Madison, WI) according to the manufacturer's recommendations. Sections were then counterstained with DAPI for the detection of nuclei. Images (five per lung section) of the lung sections were acquired with a Zeiss Imager Z1 (lens magnification, $\times 20$ ). In each image, the number of DAPI-positive cells (blue nuclei) and apoptotic cells (red) were counted manually by an investigator who was blinded to the identity of the slides (H.H.).

\section{IHC Analysis}

Immunohistochemical staining of the lung tissue was performed as previously described. ${ }^{11}$ The number of CD45-positive cells in the lung sections ( $n=3$ per group and 10 fields per lung section) were counted manually and were normalized by number of total cells in the same microscopic field.

\section{Statistical Analysis}

Results are reported as means \pm SEM. Two-way analysis of variance with Bonferroni multiple comparisons and a two-tailed, nonparametric $t$-test with Welch's correction were performed using GraphPad Prism version 6.0e (GraphPad Software, La Jolla, CA). $P<0.05$ was considered significant.

\section{Results}

RvD1 Signaling Is Altered in Human Lung Samples of COPD

To investigate whether SPM signaling pathways are disrupted in COPD in humans, we examined the expression of several key proteins involved in RvD1 signaling and metabolism. The actions of RvD1 are mediated by ALX/ FPR2 and G-protein receptor (GPR)32 (Figure 1A). The levels of expression of ALX/FPR2 and GPR32 were highly elevated in lung samples from patients with COPD compared with control lung samples (Figure 1, B-D). RvD1 is a biological substrate for inactivation by EOR (also called 15-prostaglandin dehydrogenase), ${ }^{20}$ and the level of EOR also affects the levels of other SPMs from arachidonate, such as lipoxin $\mathrm{A}_{4} \cdot{ }^{21,22}$ Control lungs showed a basal level of EOR expression, which was significantly increased in lungs with COPD (Figure 1, B and E). We also reported elsewhere that RvD1 levels were significantly decreased in bronchoalveolar lavage fluid and serum from COPD patients compared with those in healthy controls. ${ }^{23}$ These results suggest that at least some SPM signaling pathways are dysregulated in COPD.

\section{Bioactive Lipid Mediators Are Present in Mouse Lung}

Previously, we showed that treatment with RvD1 inhibited acute cigarette smoke-induced inflammation in mice. ${ }^{11}$ However, there is no information on which lipid mediators are present in the lungs of smoke-exposed and control mice. For this analysis we used lung tissue from mice exposed to cigarette smoke for 3 days, as in our acute model. ${ }^{11}$ Several important proresolving lipid mediators were identified, including RvD1, 
A

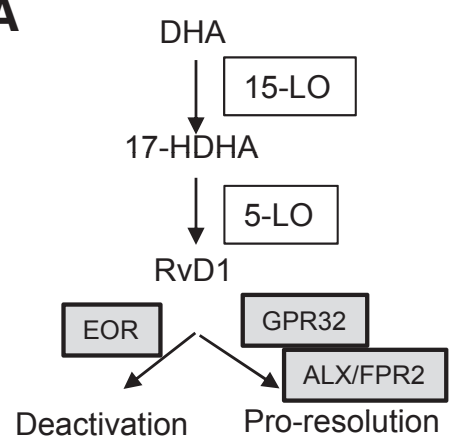

C

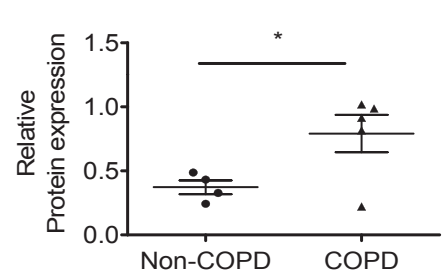

B

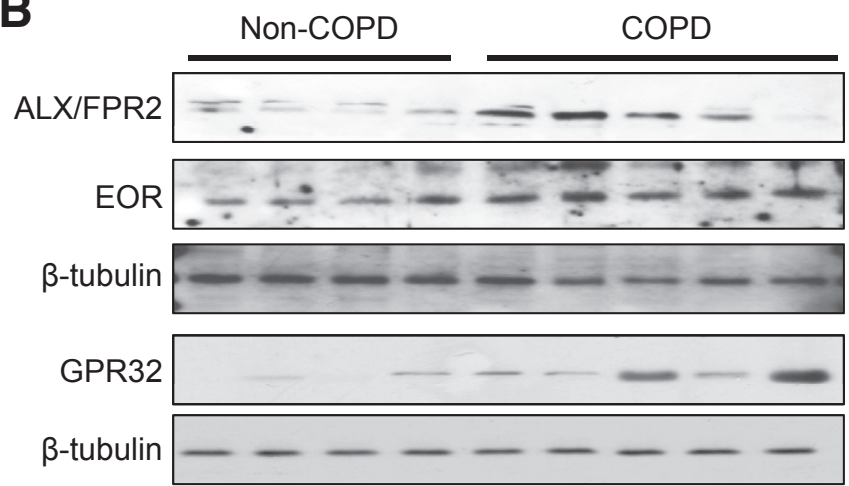

D

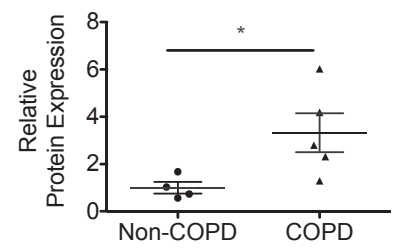

E

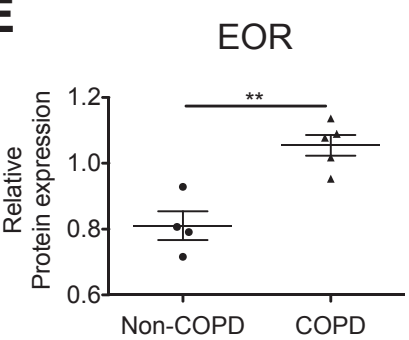

Figure 1 Proresolving signaling involving resolvin D1 (RvD1) is altered in lungs from patients with chronic obstructive pulmonary disease (COPD). A: RvD1 signaling pathway, showing synthetic and degrading enzymes and receptors. B: Levels of lipoxin $\mathrm{A}_{4}$ receptor/formyl peptide receptor 2 (ALX/FPR2), G-protein receptor (GPR)32, and eicosanoid oxidoreductase (EOR) in human lung lysates were examined by Western blot analysis. Each lane represents a lung sample from an individual donor. ALX/FPR2 and EOR were from the same blot, whereas GPR32 was from a second blot. Each individual blot was stripped and reprobed with $\beta$-tubulin as a loading control. C-E: Levels of ALX/FPR2 (C) GPR32 (D), and EOR (E) were quantitated by densitometry and normalized to $\beta$-tubulin. Data are expressed as means \pm SEM. $n=4$ (control, non-COPD lungs); $n=5$ (COPD lungs). ${ }^{*} P<0.05,{ }^{*} P<0.01$ by nonparametric, two-tailed $t$-test. 5 -L0, 5-lipoxygenase; 15-L0, 15-lipoxygenase; 17-HDHA, 17-hydroxy docosahexaenoic acid; DHA, docosahexaenoic acid.

protectin D1, and 17-hydroxydocasahexaenoic acid, a precursor to RvD1 (Supplemental Figure S1). ${ }^{9}$ Although the level of RvD1 did not change with acute cigarette smoke exposure, its presence suggests that $\mathrm{RvD} 1$ is a part of the normal homeostasis mechanism in mouse lung. Taken together, our results from human and mouse lung tissue support our hypothesis that supplementation with exogenous RvD1 could inhibit long-term cigarette smokeinduced lung disease.

\section{AT-RvD1 Inhibits Long-Term Cigarette Smoke-Induced Airspace Enlargement in Mice}

For these in vivo studies, we used the 17-R epimer of RvD1, also known as AT-RvD1, because it is resistant to inactivation by endogenous oxidoreductases and may have a longer duration of biological activity in vivo. ${ }^{11,13,21}$ We recently reported that AT-RvD1 inhibited acute cigarette smokeinduced inflammation in mice, and accelerated resolution of inflammation after smoking cessation. ${ }^{11}$ Here, we investigated whether AT-RvD1 inhibits the development of cigarette smoke-induced airspace enlargement in a well-developed mouse model. Female A/J mice were exposed to dilute mainstream cigarette smoke for 5 days/week for 12 weeks. ${ }^{24}$ Groups of mice were treated with AT-RvD1 twice a week either by inhalation or by i.v. injection as described in Materials and Methods.
As previously described, ${ }^{23}$ cigarette smoke induced airspace enlargement and parenchymal destruction, as demonstrated by histological examination and by mean linear intercept (Figure 2, A and B, respectively). Treatment with AT-RvD1, when given by both inhalation (Figure 2, A and B) and i.v. injection (Supplemental Figure S2), was associated with significantly inhibited development of airspace enlargement. With AT-RvD1, several additional lung morphometry parameters were preserved, including alveolar surface-to-volume ratio (Figure 2C), mean chord length, alveolar area, septal length, and alveolar radius (Supplemental Figure S3). Because the efficacy of ATRvD1 was similar when AT-RvD1 was given by inhalation or i.v. injection, we conducted our subsequent analyses using the inhalation group.

We also investigated the effects of long-term cigarette smoke exposure on proresolving signaling pathways. Similar to COPD patients, mice exposed to cigarette smoke long-term showed increased expression of Alx/Fpr2 and Eor (Figure 3A). We also examined the expression of murine 12/15-lipoxygenase (12/ 15Lox; an orthologue of human 15-LOX-1), a proresolving enzyme that coverts precursors into several SPMs, including RvD1. ${ }^{9}$ Long-term smoke exposure was associated with significantly reduced 12/15Lox mRNA levels (Figure 3B). Interestingly, although treatment with AT-RvD1 was associated with a significantly increased level of 12/15Lox mRNA, the expression of Alx/Fpr2 or Eor were not affected (Figure 3A). 
A

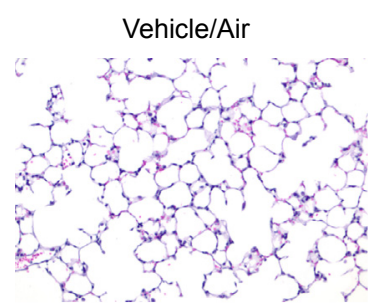

Vehicle/Smoke

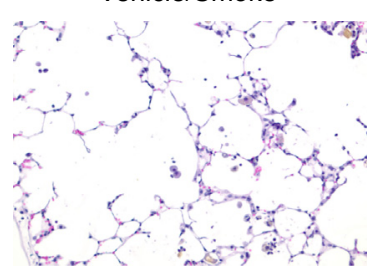

B

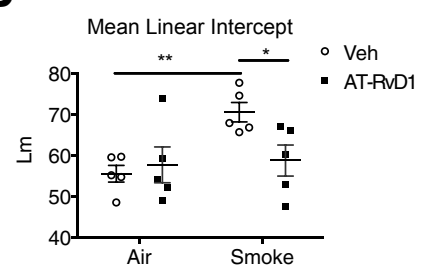

AT-RvD1/Air

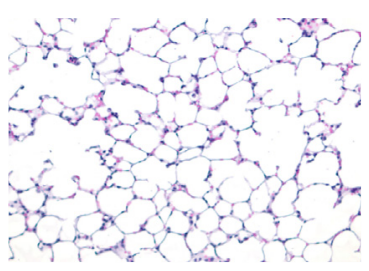

AT-RvD1/Smoke

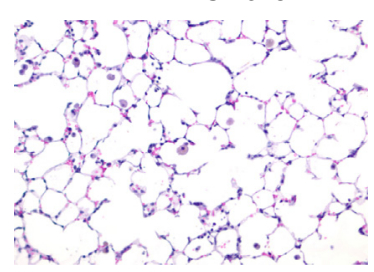

C

Surface Area/Lung Volume

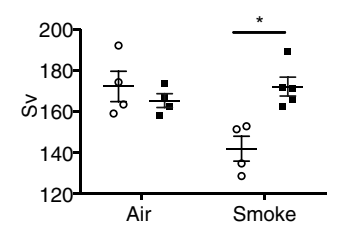

Figure 2 Aspirin-triggered resolvin D1 (AT-RvD1) attenuates long-term cigarette smoke-induced destructive airspace enlargement. A: Representative hematoxylin and eosin-stained photomicrographs of lung sections from mice subjected to 12 weeks of cigarette smoke exposure with AT-RvD1 treatment or without (Veh). B and C: Mean linear intercept (Lm) (B) and alveolar surface-to-volume ratio (Sv) (C) were determined as described in Materials and Methods. $n=5$ mice per treatment group. ${ }^{*} P<0.05$, ${ }^{*} P<0.01$ by $t$-tests with Welch's correction.

Taken together, these results suggest that chronic smoke exposure decreases the production of endogenous SPMs and increases their degradation, and that, although treatment with exogenous AT-RvD1 can prevent the airspace enlargement phenotype, it does not directly restore the endogenous SPM pathways.

\section{AT-RvD1 Inhibits Chronic Inflammation Elicited by Long-Term Cigarette Smoke Exposure}

Because chronic inflammation is a key factor in COPD, and RvD1 is known to inhibit acute lung inflammation and injury, we hypothesized that the protective effect of AT-RvD1 in long-term cigarette smoke exposure is due to the inhibition of chronic inflammation. ${ }^{25,26}$ Therefore, we investigated whether AT-RvD1 alters the accumulation of inflammatory cells in lungs exposed to cigarette smoke, using immunohistochemistry analysis, flow cytometric analysis, and quantitative PCR. Lung sections were stained with the common leukocyte antigen CD45. In long-term cigarette smoke-exposed lungs, the number of CD45positive cells in lung tissue was increased compared with that in mice exposed to ambient air. Treatment with ATRvD1 was associated with a significantly reduced total

leukocyte number in lung tissue (Figure 4, A and B). We also determined the percentages of neutrophils and macrophages in lung tissue digests by flow cytometry. Cigarette smoke can activate neutrophils and induce macrophage recruitment, and as expected, neutrophils and macrophages were increased in cigarette smoke-exposed lungs (Figure 4, C and D, Supplemental Figure S4). This increase was blunted with AT-RvD1 treatment, suggesting that both neutrophils and macrophages are potential targets of AT-RvD1.

To further understand how AT-RvD1 modulates chronic inflammation, the expression of selected classic proinflammatory genes was analyzed by quantitative PCR of wholelung RNA. In the cigarette smoke-exposed lungs, levels of Il-6, Cxcl1 (keratinocyte chemoattractant, a murine analogue of human IL-8), and $\mathrm{Ccl} 2$ (monocyte chemotactic protein 1) mRNA (Figure 4, E-G, respectively) were highly increased. With AT-RvD1, the increases in Il-6 and Cxcl1 were inhibited, suggesting that AT-RvD1 alleviates chronic lung inflammation via down-regulation of these disease-relevant genes (Figure 4, E and F). However, the expression of $\mathrm{Ccl} 2$ was increased by
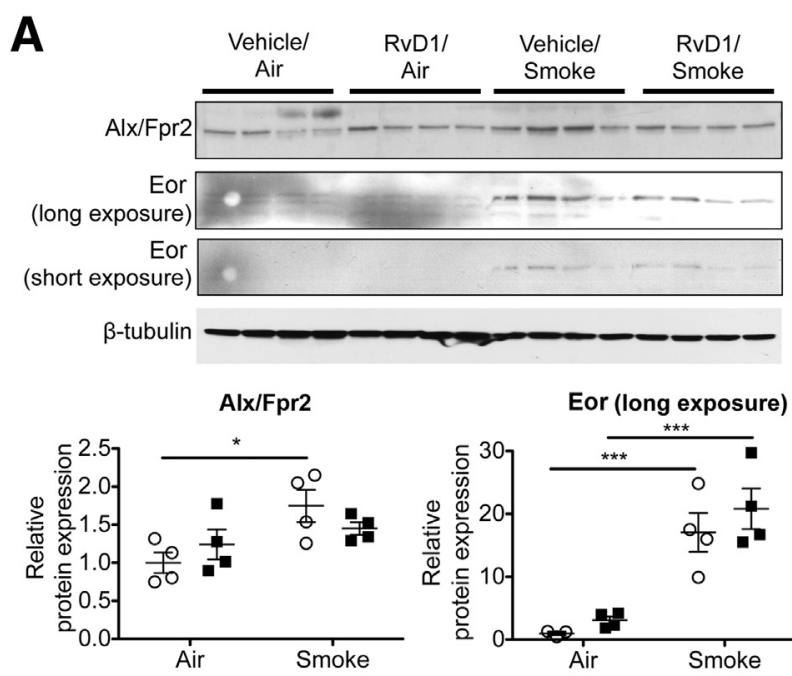

B

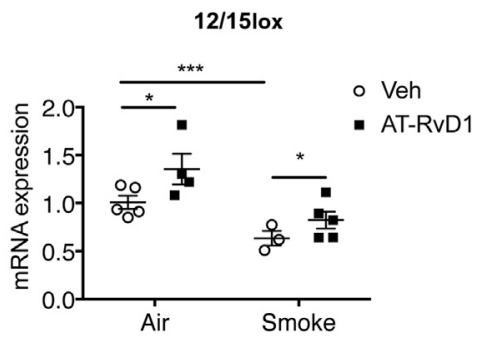

Figure 3 Cigarette smoke exposure dysregulates proresolving signaling in mouse lung. A: Whole-lung lysates were analyzed by Western blot analysis for the expression of lipoxin $\mathrm{A}_{4}$ receptor/formyl peptide receptor 2 (Alx/Fpr2) and eicosanoid oxidoreductase (Eor). $\beta$-Tubulin was used as a loading control. Original blots were cropped for illustration purposes. The results were quantitated by densitometry and normalized to $\beta$-tubulin. B: 12/15-Lipoxygenase (12/15Lox) mRNA was determined in whole-lung RNA by real-time PCR. Data are expressed as means \pm SEM. $n=3$ to 5 mice per group. ${ }^{*} P<0.05,{ }^{* * *} P<0.001$ by two-way analysis of variance. RvD1, resolvin D1; Veh, vehicle. 
A

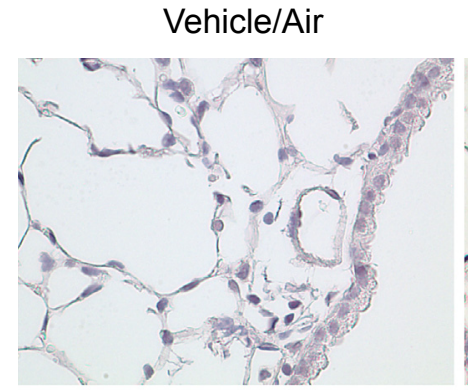

Vehicle/Smoke

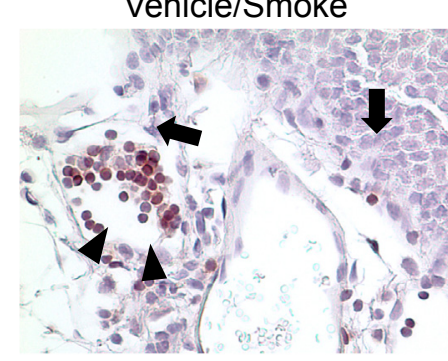

C

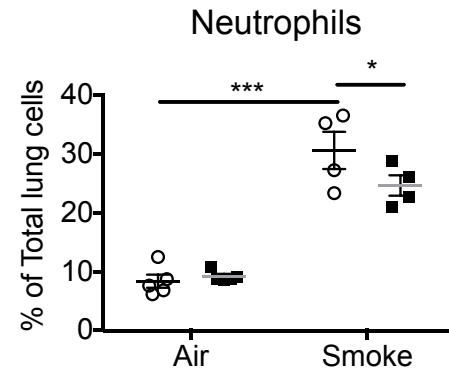

F

$\mathrm{Cxcl} 1 / \mathrm{Kc}$

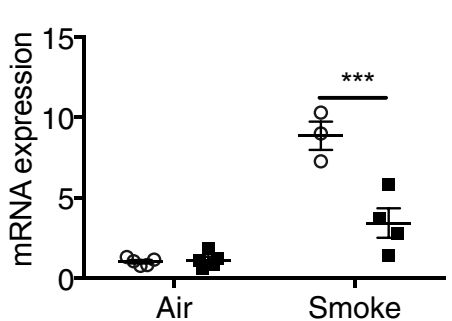

AT-RvD1/Air

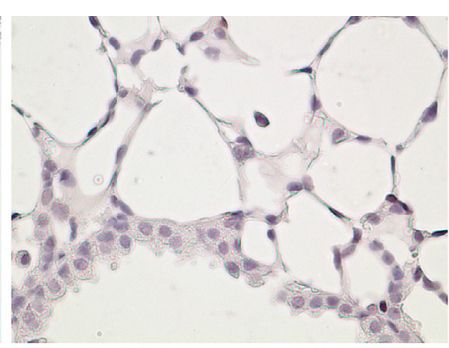

AT-RvD1/Smoke

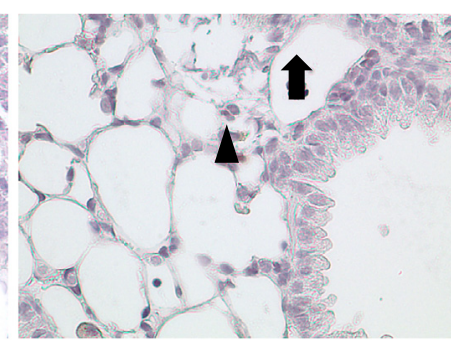

D

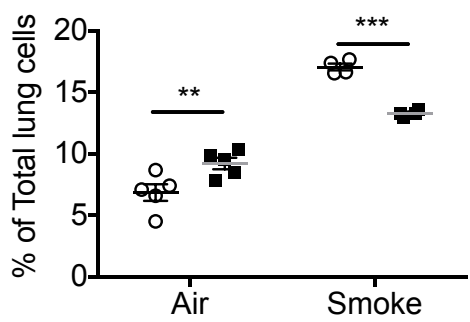

G

Ccl2/Mcp-1

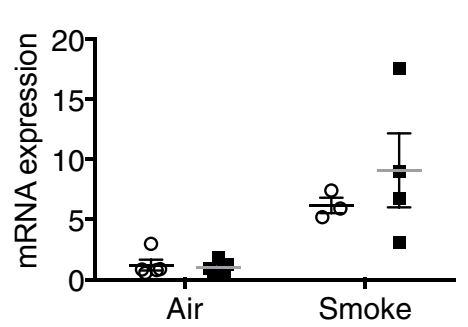

B

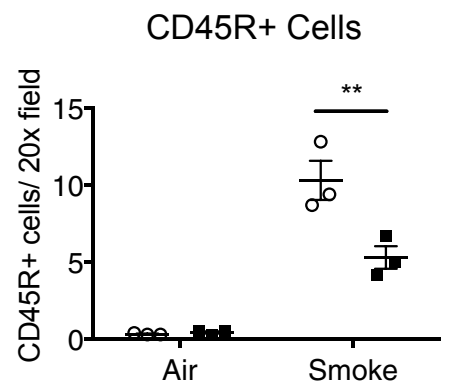

E

II-6

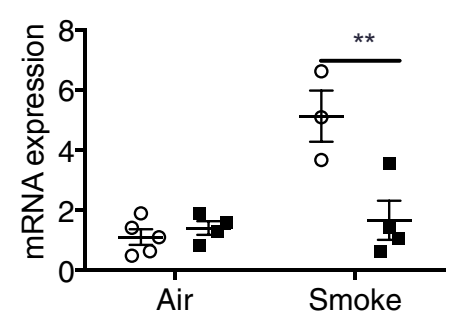

H

II-10

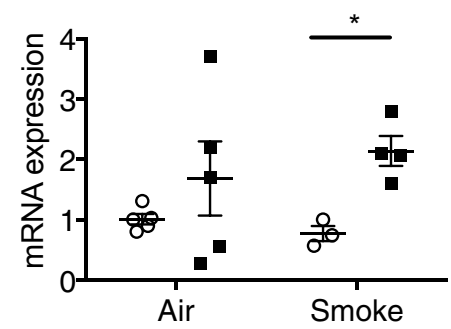

I
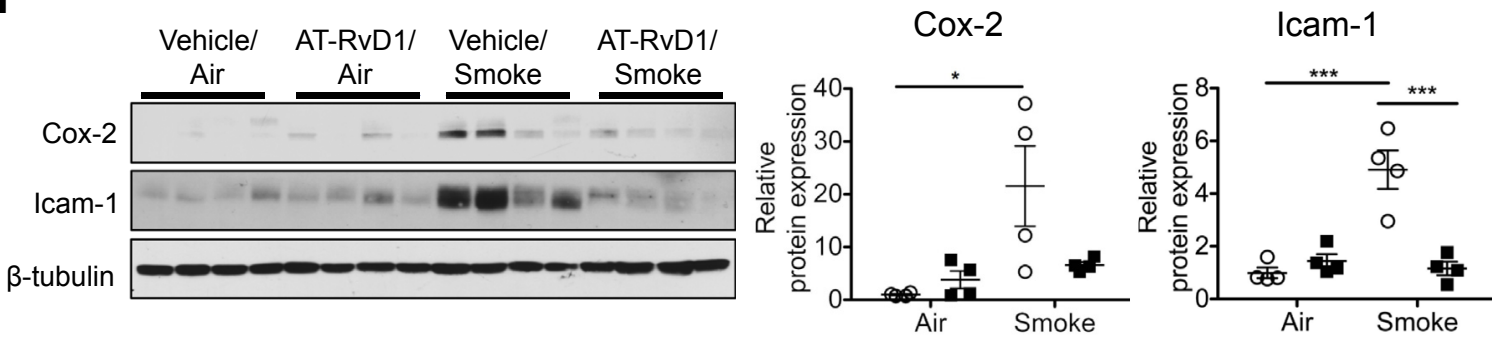

Figure 4 Aspirin-triggered resolvin D1 (AT-RvD1) reduces chronic inflammation elicited by chronic cigarette smoke. A: Representative photomicrographs of CD45-stained lung sections after exposure to air or cigarette smoke, treated with vehicle (closed squares) or AT-RvD1 (open circles). The arrow indicates $\mathrm{CD}_{4} 5^{+}$cells in peribronchial and perivascular spaces; arrowhead, accumulated $\mathrm{CD} 45^{+}$cells in the interstitial spaces. B: $\mathrm{CD}_{4} 5^{+}$cells were counted in 5 highpower fields per mouse. $\mathbf{C}$ and $\mathbf{D}$ : Percentages of $\mathrm{Gr}-1^{+}$neutrophils $(\mathbf{C})$ and $\mathrm{F} 4 / 80^{+}$macrophages (D) were determined by flow cytometry. E-H: $m$ RNA levels of Il-6 (E), chemokine (C-X-C motif) ligand 1/keratinocyte chemoattractant $(\mathrm{Cxcl1} / \mathrm{KC})(\mathbf{F})$, monocyte chemotactic protein 1 (MCP1/Ccl2) (G), and Il-10 (H) were quantified in whole-lung homogenates by real-time PCR. I: Levels of cyclooxygenase (Cox)-2 and intercellular adhesion molecule (Icam)-1 in mouse lung were determined by Western blot analysis. $\beta$-Tubulin was used as a loading control. The results were quantitated by densitometry and normalized to $\beta$-tubulin. Data are expressed as means \pm SEM. $n=4$ mice per group $(\mathbf{F}, \mathbf{G}$, and $\mathbf{I}) ; n=5$ mice per group $(\mathbf{B}-\mathbf{E}$ and $\mathbf{H}) .{ }^{*} P<0.05,{ }^{* *} P<0.01$, and ${ }^{* * *} P<0.001$ by two-way analysis of variance with Bonferroni post-test. 

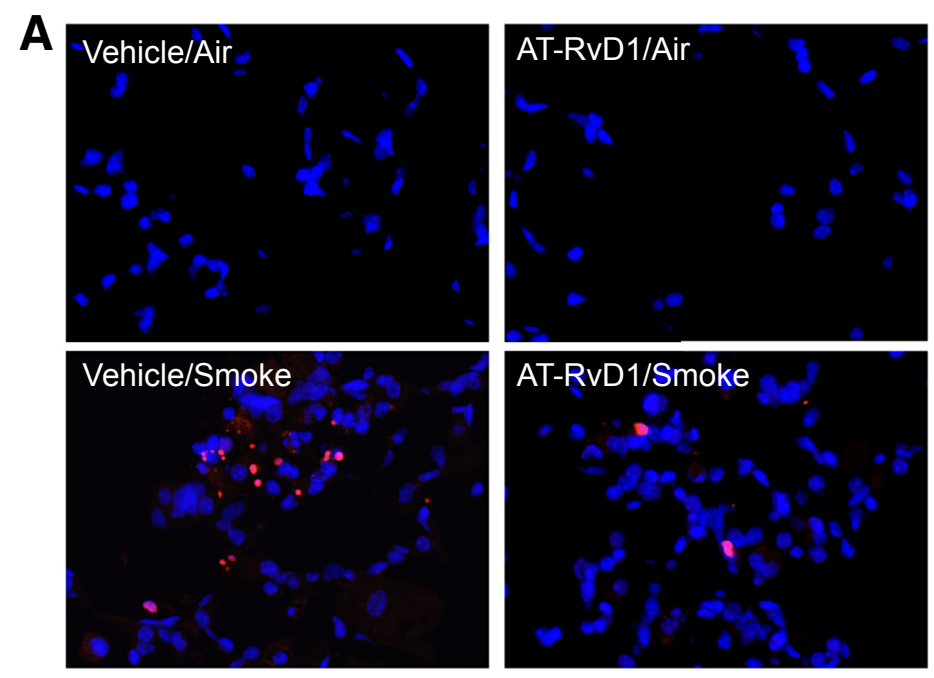

\section{B}
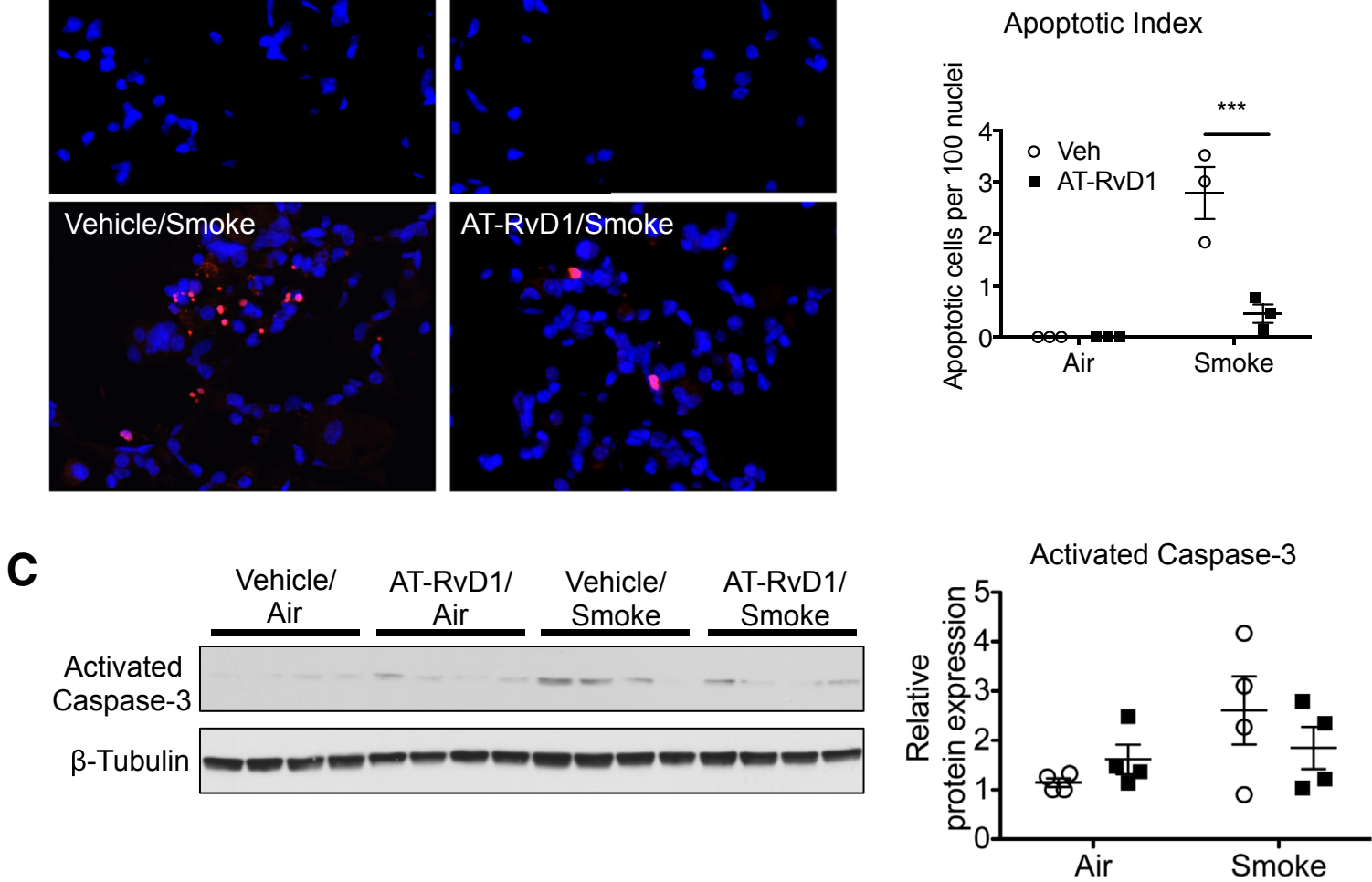

Figure 5 Aspirin-triggered resolvin D1 (AT-RvD1) reduces cigarette smoke-induced cell death. A: Representative images of lung sections stained using terminal deoxynucleotidyl transferase-mediated dUTP nick-end labeling (TUNEL). The nuclei of TUNEL-positive cells were labeled in red. Tissue sections were counterstained with DAPI to visualize cell nuclei (blue nuclei). B: TUNEL-positive cells and DAPI-stained nuclei were counted in five high-power fields per mouse. The apoptotic index was defined as the number of TUNEL-positive cells per 100 DAPI-stained nuclei. C: Level of cleaved caspase-3 was examined by Western blot analysis. The results were quantitated by densitometry and normalized to $\beta$-tubulin. Data are expressed as means \pm SEM. $n=5$ mice per group (B). ${ }^{* * * P}<0.001$ for smoke/vehicle versus smoke/AT-RvD1 by two-way analysis of variance with Bonferroni post-test (B). Veh, vehicle.

cigarette smoke but was not reduced with AT-RvD1 treatment (Figure 4G). We also examined the expression of Il-10, a cytokine that has potent proresolving properties. ${ }^{5,27}$ The expression of Il-10 was significantly up-regulated with AT-RvD1, indicating that Il-10 is a target (Figure 4H). Finally, we examined expression of Cox-2, a classic proinflammatory enzyme, and intercellular adhesion molecule (Icam-1), an adhesion molecule that contributes to neutrophil and macrophage extravasation. The levels of expression of Cox-2 and Icam- 1 have been reported to be correlated with the pathogenesis of COPD. ${ }^{5}$ Consistent with our other results, these proteins were up-regulated with cigarette smoke exposure, and this increase was blocked with treatment with AT-RvD1 (Figure 4I).

\section{AT-RvD1 Attenuates Cigarette Smoke-Induced Apoptosis in Mouse Lung}

Cell death is a consequence of long-term cigarette smoke exposure and a hallmark of emphysema. ${ }^{4,28}$ Because ATRvD1-treated animals were protected from cigarette smoke-induced tissue destruction and cell inflammation, we also examined the effect of AT-RvD1 on lung cell death Cigarette smoke-exposed lungs were assessed by TUNEL staining, a marker of DNA damage associated with cell death. Because long-term smoke exposure is known to induce cell death, it was not surprising that the number of TUNEL-positive cells was significantly increased in the lungs that were exposed to long-term cigarette smoke (Figure 5, A and B). ${ }^{29-31}$ AT-RvD1 was associated with reduced TUNEL staining induced by cigarette smoke, suggesting that the reduction of airspace destruction by AT-RvD1 may be a consequence of reduced cell death. To further the effect of AT-RvD1 on apoptosis pathways, we examined the expression of activated caspase-3, a proapoptotic factor that is induced by cigarette smoke and is increased in lungs from patients with COPD. ${ }^{4,32}$ Caspase- 3 showed a nonsignificant trend toward decrease with AT-RvD1 treatment (Figure 5C).

\section{AT-RvD1 Ameliorates Long-Term Cigarette Smoke-Induced 0xidative Stress}

Oxidative stress in COPD can come from both cigarette smoke as well as activated inflammatory cells., ${ }^{43}$ To 
A

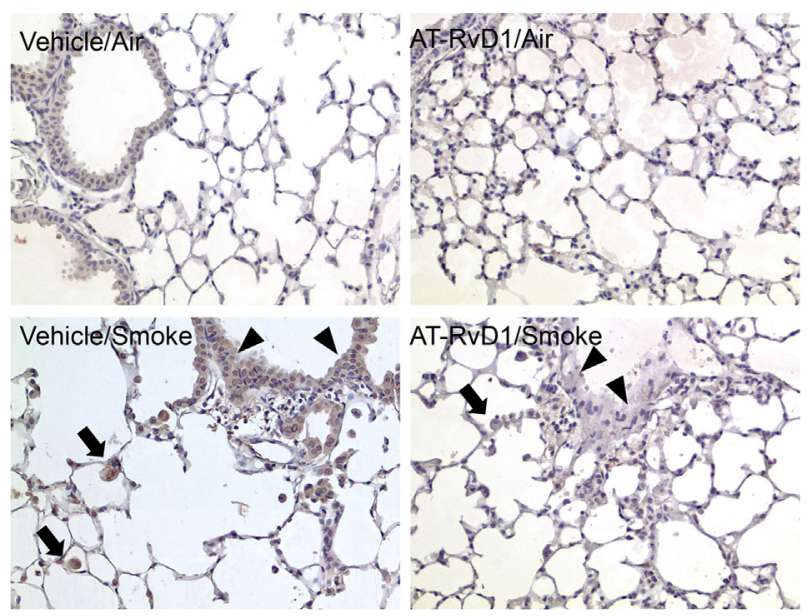

B

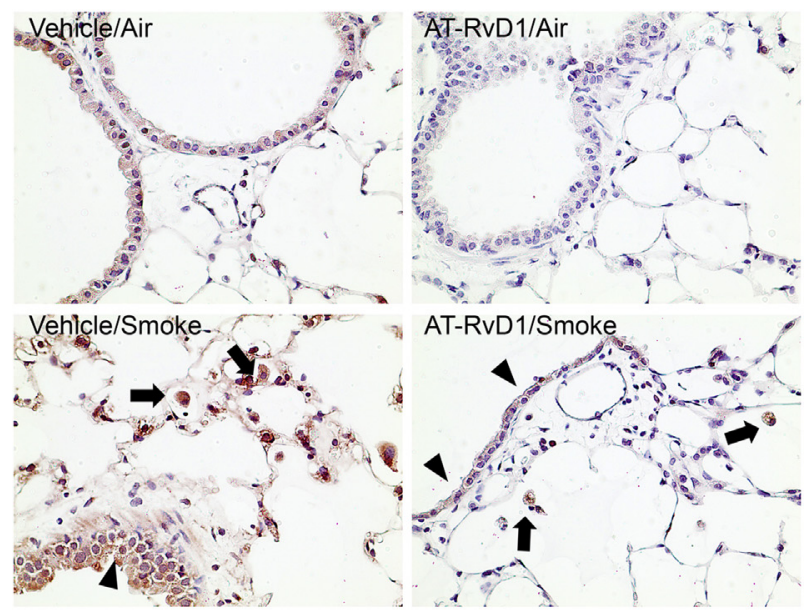

C

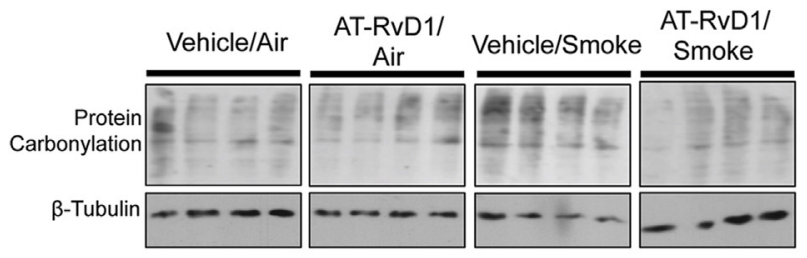

O Veh

- AT-RvD1

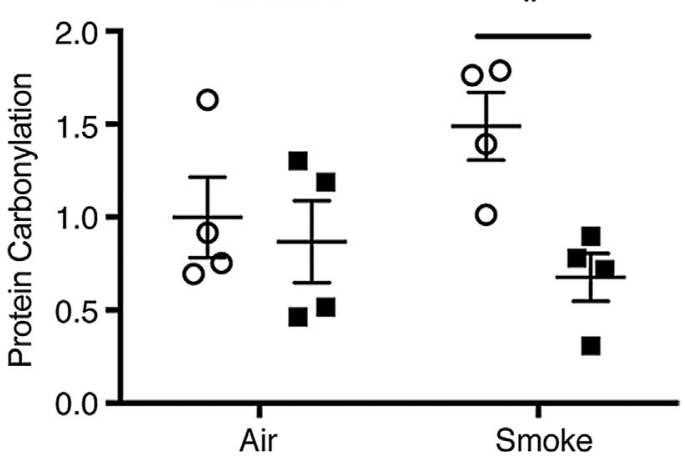

examine the effect of AT-RvD1 on oxidative stress, lung sections were stained for nitrotyrosine and $8-\mathrm{OHdG}$, markers of protein nitrosylation by reactive nitrogen species, and DNA oxidation by reactive oxygen species. Both nitrotyrosine and $8-\mathrm{OHdG}$ were increased with long-term cigarette smoke exposure (Figure 6, A and B). Importantly, in AT-RvD1-treated animals, not only was the amount of staining reduced but also the number of inflammatory cells stained with $8-\mathrm{OHdG}$ and nitrotyrosine was reduced (Figure 6, A and B). We also analyzed protein carbonylation by Western blot analysis. Consistent with earlier findings, the level of protein carbonylation was increased with cigarette smoke exposure, and this increase was alleviated with AT-RvD1 treatment (Figure 6C).

\section{AT-RvD1 Treatment Does Not Promote a Macrophage M2 Phenotype}

We recently reported that AT-RvD1 promoted differentiation of M2 macrophages in mice exposed acutely (3 days) to cigarette smoke. ${ }^{11}$ Although M2 macrophages are believed to be beneficial in mitigating inflammation and promoting resolution, ${ }^{34,35}$ there is evidence to suggest that M2 macrophages also promote fibrosis in long-term settings. ${ }^{36-38}$ To evaluate whether AT-RvD1 promotes M2 macrophage polarization in our model of long-term cigarette smoke exposure, lung macrophages were isolated from whole-lung digests by cell sorting, and the levels of expression of M1 and M2 markers were quantitated using real-time PCR. In air-exposed mice, macrophages from mice treated with AT-RvD1 exhibited increased RNA for the M2 marker mannose receptor Mrc1 (mannose receptor, CD206) and decreased RNA for the M1 marker inducible nitric oxide synthase (Figure 7, A-D). However, the M1 and M2 profiles of lung macrophages from mice exposed to cigarette smoke and treated with AT-RvD1 were similar. We also evaluated collagen deposition by staining lung sections with Gomori's trichrome. With AT-RvD1, changes associated with fibrosis, such as interstitial deposition of collagen fibrils, were not promoted in the presence or absence of long-term cigarette smoke exposure (Figure 7E).

Figure 6 Aspirin-triggered resolvin D1 (AT-RvD1)-treated lung shows a reduction in oxidative stress. A: Mice were exposed to air or cigarette smoke and treated with vehicle or AT-RvD1, and lung sections were stained for anti-8-hydroxydeoxyguanosine (OHdG). $8-0 \mathrm{HdG}$ staining (brown) is evident in smoke-exposed mice in both epithelium (arrowheads) and macrophages (arrows), and is reduced with treatment with AT-RVD1. B: Lung sections from smoke-exposed and AT-RvD1-treated mice were stained for nitrotyrosine (red). Nitrotyrosine staining is broadly distributed in lung sections from smoke-exposed and vehicle-treated mice (Veh), especially in the epithelium (arrowheads) and macrophages (arrows), and is reduced with treatment with AT-RvD1. C: Protein carbonylation was measured in lung homogenates using an 0xyBlot kit following the manufacturer's instructions (EMD Millipore, Billerica, MA). The same blot was stripped and reprobed with $\beta$-tubulin as a loading control. Each lane represents an individual mouse. Protein carbonylation was quantified by densitometry normalized to $\beta$-tubulin. Data are expressed as means $\pm \mathrm{SEM}$. ${ }^{*} P<0.05$ versus vehicle/smoke. 
A
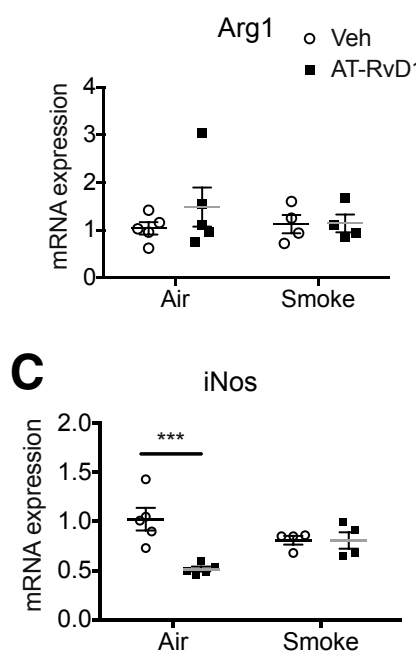

B
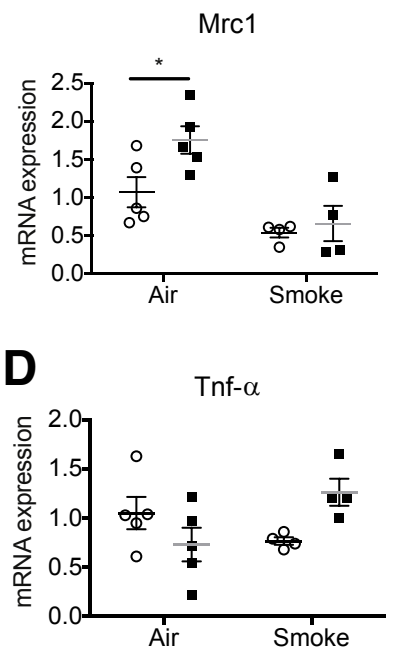

E
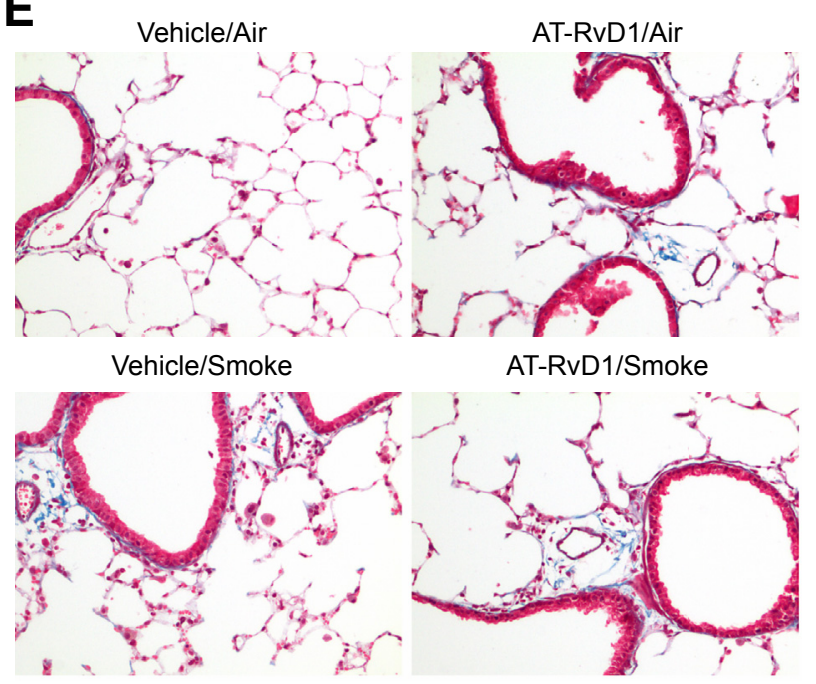

Figure 7 Aspirin-triggered resolvin D1 (AT-RvD1) neither affects macrophage phenotype nor induces fibrosis. A-D: Total RNA was isolated from lung F4/80 ${ }^{+}$ macrophages and subjected to quantitative PCR to determine the levels of $\operatorname{Arg} 1(\mathbf{A})$, mannose receptor (MrC)-1 (B), inducible nitric oxide synthase (iNos; C), and tumor necrosis factor (Tnf)- $\alpha$ (D). 18sRNA was used as an internal control. E: Representative photomicrographs of lungs exposed to chronic smoking, with or without AT-RvD1 treatment, and stained for Gomori's trichrome stain. Collagen deposition was stained blue. Sections were counterstained with hematoxylin for nuclei. Data are expressed as means \pm SEM. $n=4$ or 5 mice per group. ${ }^{*} P<0.05,{ }^{* *} P<0.001$ versus vehicle/air by two-way analysis of variance with Bonferroni post-test.

\section{Discussion}

Chronic inflammation is a major component of COPD. It has long been thought that COPD is marked by defective control of inflammation, but the reasons had not been fully elucidated. ${ }^{4,5}$ Here, we show for the first time that some proresolution pathways are dysregulated both in COPD patients and in a mouse model of long-term cigarette smoke exposure, with increased levels of RvD1 receptors but also increased expression of an RvD1-degrading enzyme (Figures 1 and 3). We also show for the first time that supplementation with AT-RvD1 not only inhibits emphysema but also attenuates chronic cellular inflammation, oxidative stress, and cell death, all of which are key in the pathogenesis of COPD (Figures 2, 4, 5, and 6 and Supplemental Figures S2, S3, and S4).

Although it has previously been reported that ALX/FPR2 is up-regulated in COPD, ${ }^{39,40}$ we show here for the first time that GPR32 and EOR are also up-regulated in lung tissue from COPD patients, and that Eor is up-regulated and 12/ 15 Lox is down-regulated in mice exposed to cigarette smoke long-term. These findings support that long-term tobacco smoke exposure affects a significant proresolution pathway shared by humans and mice. EOR was initially thought to degrade proinflammatory mediators such as prostaglandin $\mathrm{E}(\mathrm{PGE})_{2}$ to reduce inflammatory responses, ${ }^{41}$ but it is now known to also degrade proresolving mediators including $\mathrm{LXA}_{4}$ and $\mathrm{RvD} 1{ }^{21,22} \mathrm{PGE}_{2}$ also plays a proresolving role by activating lipid class switching. ${ }^{42}$ Increased EOR likely inhibits resolution by degrading proresolving mediators and by dampening $\mathrm{PGE}_{2}$-driven lipid class switching. Ligands for ALX/FPR2 include the proresolving mediators $\mathrm{LXA}_{4}$ and
$\mathrm{RvD1}$, and also the proinflammatory molecule serum amyloid A. ${ }^{43}$ (Human GPR32 has no other known ligands.) The effects of ligand binding to ALX/FPR2 are concentration, conformation, and context specific. ${ }^{44}$ Levels of $\mathrm{LXA}_{4}$ and RvD1 are reduced in sputum, serum, and exhaled breath from COPD patients and serum amyloid A is increased. ${ }^{43,45,46}$ Thus, upregulation of ALX/FPR2 expression, in combination with increased EOR and decreased $\mathrm{LXA}_{4}$ and RvD1, could lead to increased proinflammatory signaling by serum amyloid A due to a loss of competition for receptor binding by proresolving molecules. Finally, we have also identified multiple differences in SPMs in human exhaled breath condensate between healthy volunteers and COPD patients, and demonstrated a significant decrease in RvD1 in both bronchoalveolar lavage fluid and serum from COPD patients. ${ }^{23}$ Taken together, we suggest that these data reflect an attempt to limit inflammation by upregulating the receptors for proresolving mediators that is defeated by increased degradation of those mediators.

COPD is a complex inflammatory disease that includes chronic infiltration of the lungs by inflammatory cells. ${ }^{5}$ In this study, we have demonstrated that cigarette smoke-exposed lungs had increased macrophages and neutrophils, and that these increases were attenuated by AT-RvD1 (Figure 4). Inflammatory cells are major sources of proinflammatory mediators that amplify and propagate inflammatory responses. 5 It is known that proinflammatory mediators, including IL-6, IL-8, and monocyte chemotactic protein 1, play crucial roles in orchestrating immune responses by regulating the trafficking of inflammatory and immune cells to the lung in response to cigarette smoke exposure. ${ }^{5}$ In contrast, IL-10 is a profoundly anti-inflammatory cytokine that is decreased in COPD and other chronic lung inflammatory diseases, ${ }^{47}$ and is up-regulated 
by RvD1. ${ }^{11,48}$ Previously, we reported that RvD1 attenuated the production of the neutrophil chemoattractant IL-8 by human lung epithelial cells by inhibiting the activation of NF- $\kappa B$ and mitogen-activated protein kinase. ${ }^{49} \mathrm{RvD} 1$ also directly inhibits neutrophil chemotaxis. ${ }^{21,50}$ Here, we found that AT-RvD1 dramatically altered the inflammatory environment in cigarette smoke-exposed lungs, with reductions in total inflammatory cells, neutrophils, and the expression of proinflammatory cytokines, Cox-2, and ICAM-1, and increased expression of IL-10. We hypothesize that the reduction in inflammation results from both the direct inhibition of neutrophil chemotaxis and activation as well as inhibition of proinflammatory signaling by epithelial and other lung cells, resulting in decreased levels of expression of chemotactic factors and adhesion molecules. The specific mechanism of action of AT-RvD1 on inflammatory cells is not fully understood at this time; however, we recently reported that RvD1 inhibits the release of proinflammatory signals by lung epithelial cells by blocking the NF- $\kappa \mathrm{B}$ and ERK pathways. ${ }^{49}$ AT-RvD1 may also interfere with the proinflammatory effects of serum amyloid A binding to ALX/FPR2.

Although AT-RvD1 reduced the number of tissue macrophages in smoke-exposed mice, we suspected that it might also alter their phenotype. We and others have reported that RvD1 promotes alternative activation of macrophages (the M2 phenotype) ${ }^{11,50}$ and that the M2 macrophages appear to mitigate inflammation and promote the resolution of inflammation in acute models and cell culture systems. ${ }^{11,51}$ However, some evidence suggests that in chronic diseases, M2 macrophages may promote tissue remodeling and repair, leading to fibrosis. ${ }^{38}$ In our model of long-term smoke exposure with AT-RvD1 treatment, we did not find evidence of preferential M1 or M2 activation. We hypothesize that a strong M2 phenotype may be a feature of early activation of resolution pathways in acute models, whereas long-term AT-RvD1 treatment results in a more balanced macrophage phenotype. Importantly, there were no signs of tissue fibrosis with AT-RvD1 treatment.

Concurrent with the reduction in cellular inflammation, ATRvD1 treatment also was associated with reduced oxidative stress and cell death. Cigarette smoke contains high levels of free radicals and free radical-generating compounds that contribute to oxidative stress. ${ }^{7}$ However, it is also clear that a second significant source of oxidative stress in chronic lung disease including COPD are inflammatory cells themselves., Cigarette smoke and oxidative stress are both strong stimulators of cell death and are key factors in the tissue destruction seen in emphysema. ${ }^{4,28,30}$ RvD1 itself is not an antioxidant at the concentrations used, ${ }^{52}$ so it is possible that reduced oxidative stress and cell death is an indirect effect of a reduction in the numbers of inflammatory cells. Alternatively, recent evidence suggests that RvD1 has direct antiapoptotic effects. RvD1 inhibits apoptosis caused by antibiotic-induced ER stress in liver cells ${ }^{53}$ and also attenuates cigarette smoke-induced cell death in murine macrophages by upregulating the expression of the antiapoptotic proteins B-cell lymphoma-2 and B-cell lymphoma-extra large. ${ }^{54}$ Taken together, we suspect that the net reductions in oxidative stress and cell death are likely due to a combination of direct effects of RvD1 on apoptosis signaling pathways as well as reductions in the numbers of inflammatory cells.

Corticosteroids, currently used for controlling the symptoms of COPD exacerbations, also block the production of proinflammatory mediators, which may ultimately contribute to an enhanced inflammatory pathology. ${ }^{9,55}$ There is also increasing thought that therapies that target a single mediator or cascade are unlikely to provide major clinical benefits in patients with COPD because COPD is a complex inflammatory disease involving multiple pathological pathways and mediators. ${ }^{56}$ RvD1 and other SPMs are naturally occurring molecules that act to ensure tissue homeostasis by modulating the phenotypes of a wide variety of cell types and by targeting multiple signaling pathways. ${ }^{9}$ Our study provides insight into the use of this new genus of proresolution mediators as therapeutic reagents that can dampen pathological signaling pathways in chronic inflammation and reinforce proresolving pathways that promote a return to homeostasis.

\section{Acknowledgments}

We thank Drs. Tirumalai Rangasamy and Rubin M. Tuder for providing MetaMorph Microscopy Automation and Image Analysis software for mouse lung morphometric analysis, Wade Narrow and Thad Vickery (BWH, CNS laboratory) for expert technical assistance, and Kristina M. Owens for help in writing the manuscript.

H.-M.H., T.H.T., C.N.S., R.P.P., and P.J.S. conceived the study and designed the experiments. H.-M.H., T.H.T., and R.A.C. performed experiments and collected data. H.-M.H., T.H.T., R.A.C., C.N.S., R.P.P., and P.J.S. analyzed the data and wrote the manuscript.

\section{Supplemental Data}

Supplemental material for this article can be found at http://dx.doi.org/10.1016/j.ajpath.2015.08.008.

\section{References}

1. Yoshida T, Tuder RM: Pathobiology of cigarette smoke-induced chronic obstructive pulmonary disease. Physiol Rev 2007, 87: 1047-1082

2. Salvi S, Barnes PJ: Is exposure to biomass smoke the biggest risk factor for COPD globally? Chest 2010, 138:3-6

3. Barnes PJ: New therapies for chronic obstructive pulmonary disease. Med Princ Pract 2010, 19:330-338

4. Tuder RM, Petrache I: Pathogenesis of chronic obstructive pulmonary disease. J Clin Invest 2012, 122:2749-2755

5. Barnes PJ: Mediators of chronic obstructive pulmonary disease. Pharmacol Rev 2004, 56:515-548

6. Hogg JC, Chu F, Utokaparch S, Woods R, Elliott WM, Buzatu L, Cherniack RM, Rogers RM, Sciurba FC, Coxson HO, Pare PD: The 
nature of small-airway obstruction in chronic obstructive pulmonary disease. N Engl J Med 2004, 350:2645-2653

7. Zuo L, He F, Sergakis GG, Koozehchian MS, Stimpfl JN, Rong Y, Diaz PT, Best TM: Interrelated role of cigarette smoking, oxidative stress, and immune response in COPD and corresponding treatments. Am J Physiol Lung Cell Mol Physiol 2014, 307:L205-L218

8. Holguin F: Oxidative stress in airway diseases. Ann Am Thorac Soc 2013;10(Suppl):S150-S157

9. Serhan CN: Pro-resolving lipid mediators are leads for resolution physiology. Nature 2014, 510:92-101

10. Shahar E, Folsom AR, Melnick SL, Tockman MS, Comstock GW, Gennaro V, Higgins MW, Sorlie PD, Ko WJ, Szklo M; Atherosclerosis Risk in Communities Study Investigators: Dietary n-3 polyunsaturated fatty acids and smoking-related chronic obstructive pulmonary disease. N Engl J Med 1994, 331:228-233

11. Hsiao HM, Sapinoro RE, Thatcher TH, Croasdell A, Levy EP, Fulton RA, Olsen KC, Pollock SJ, Serhan CN, Phipps RP, Sime PJ: A novel anti-inflammatory and pro-resolving role for resolvin D1 in acute cigarette smoke-induced lung inflammation. PLoS One 2013, 8 : e58258

12. Wang B, Gong X, Wan JY, Zhang L, Zhang Z, Li HZ, Min S: Resolvin D1 protects mice from LPS-induced acute lung injury. Pulm Pharmacol Ther 2011, 24:434-441

13. Rogerio AP, Haworth O, Croze R, Oh SF, Uddin M, Carlo T, Pfeffer MA, Priluck R, Serhan CN, Levy BD: Resolvin D1 and aspirin-triggered resolvin D1 promote resolution of allergic airways responses. J Immunol 2012, 189:1983-1991

14. Dalli J, Serhan CN: Specific lipid mediator signatures of human phagocytes: microparticles stimulate macrophage efferocytosis and pro-resolving mediators. Blood 2012, 120:e60-e72

15. Colas RA, Shinohara M, Dalli J, Chiang N, Serhan CN: Identification and signature profiles for pro-resolving and inflammatory lipid mediators in human tissue. Am J Physiol Cell Physiol 2014, 307: C39-C54

16. Thatcher TH, Maggirwar SB, Baglole CJ, Lakatos HF, Gasiewicz TA, Phipps RP, Sime PJ: Aryl hydrocarbon receptordeficient mice develop heightened inflammatory responses to cigarette smoke and endotoxin associated with rapid loss of the nuclear factor-kappaB component RelB. Am J Pathol 2007, 170:855-864

17. Lakatos HF, Burgess HA, Thatcher TH, Redonnet MR, Hernady E, Williams JP, Sime PJ: Oropharyngeal aspiration of a silica suspension produces a superior model of silicosis in the mouse when compared to intratracheal instillation. Exp Lung Res 2006, 32:181-199

18. Kawakami M, Paul JL, Thurlbeck WM: The effect of age on lung structure in male BALB/cNNia inbred mice. Am J Anat 1984, 170: $1-21$

19. Yoshida T, Mett I, Bhunia AK, Bowman J, Perez M, Zhang L, Gandjeva A, Zhen L, Chukwueke U, Mao T, Richter A, Brown E, Ashush H, Notkin N, Gelfand A, Thimmulappa RK, Rangasamy T, Sussan T, Cosgrove G, Mouded M, Shapiro SD, Petrache I, Biswal S, Feinstein E, Tuder RM: Rtp801, a suppressor of mTOR signaling, is an essential mediator of cigarette smoke-induced pulmonary injury and emphysema. Nat Med 2010, 16:767-773

20. Clish CB, Levy BD, Chiang N, Tai HH, Serhan CN: Oxidoreductases in lipoxin A4 metabolic inactivation: a novel role for 15-onoprostaglandin 13-reductase/leukotriene B4 12-hydroxydehydrogenase in inflammation. J Biol Chem 2000, 275:25372-25380

21. Sun YP, Oh SF, Uddin J, Yang R, Gotlinger K, Campbell E, Colgan SP, Petasis NA, Serhan CN: Resolvin D1 and its aspirintriggered $17 \mathrm{R}$ epimer. Stereochemical assignments, anti-inflammatory properties, and enzymatic inactivation. J Biol Chem 2007, 282: 9323-9334

22. Romano M: Lipid mediators: lipoxin and aspirin-triggered 15-epilipoxins. Inflamm Allergy Drug Targets 2006, 5:81-90

23. Croasdell A, Thatcher TH, Kottmann RM, Colas RA, Dalli J, Serhan CN, Sime PJ, Phipps RP: Resolvins attenuate inflammation and promote resolution in cigarette smoke-exposed human macrophages. Am J Physiol Lung Cell Mol Physiol 2015, 309:L888-L901

24. March TH, Wilder JA, Esparza DC, Cossey PY, Blair LF, Herrera LK, McDonald JD, Campen MJ, Mauderly JL, Seagrave J: Modulators of cigarette smoke-induced pulmonary emphysema in A/J mice. Toxicol Sci 2006, 92:545-559

25. Stockley RA, Mannino D, Barnes PJ: Burden and pathogenesis of chronic obstructive pulmonary disease. Proc Am Thorac Soc 2009, 6: $524-526$

26. Decramer M, Janssens W, Miravitlles M: Chronic obstructive pulmonary disease. Lancet 2012, 379:1341-1351

27. Barnes PJ: Novel approaches and targets for treatment of chronic obstructive pulmonary disease. Am J Respir Crit Care Med 1999, 160:S72-S79

28. Morse D, Rosas IO: Tobacco smoke-induced lung fibrosis and emphysema. Annu Rev Physiol 2014, 76:493-513

29. Rangasamy T, Misra V, Zhen L, Tankersley CG, Tuder RM, Biswal S: Cigarette smoke-induced emphysema in $\mathrm{A} / \mathrm{J}$ mice is associated with pulmonary oxidative stress, apoptosis of lung cells, and global alterations in gene expression. Am J Physiol Lung Cell Mol Physiol 2009, 296:L888-L900

30. Baglole CJ, Bushinsky SM, Garcia TM, Kode A, Rahman I, Sime PJ, Phipps RP: Differential induction of apoptosis by cigarette smoke extract in primary human lung fibroblast strains: implications for emphysema. Am J Physiol Lung Cell Mol Physiol 2006, 291:L19-L29

31. Podowski M, Calvi C, Metzger S, Misono K, Poonyagariyagorn H, Lopez-Mercado A, Ku T, Lauer T, McGrath-Morrow S, Berger A, Cheadle C, Tuder R, Dietz HC, Mitzner W, Wise R, Neptune E: Angiotensin receptor blockade attenuates cigarette smoke-induced lung injury and rescues lung architecture in mice. J Clin Invest 2012, 122:229-240

32. Zeng H, Kong X, Peng H, Chen Y, Cai S, Luo H, Chen P: Apoptosis and $\mathrm{Bcl}-2$ family proteins, taken to chronic obstructive pulmonary disease. Eur Rev Med Pharmacol Sci 2012, 16:711-727

33. Rahman I, van Schadewijk AA, Crowther AJ, Hiemstra PS, Stolk J, MacNee W, De Boer WI: 4-Hydroxy-2-nonenal, a specific lipid peroxidation product, is elevated in lungs of patients with chronic obstructive pulmonary disease. Am J Respir Crit Care Med 2002, 166:490-495

34. Martinez FO, Helming L, Gordon S: Alternative activation of macrophages: an immunologic functional perspective. Annu Rev Immunol 2009, 27:451-483

35. Gordon S: Alternative activation of macrophages. Nat Rev Immunol 2003, 3:23-35

36. Li D, Guabiraba R, Besnard AG, Komai-Koma M, Jabir MS, Zhang L, Graham GJ, Kurowska-Stolarska M, Liew FY, McSharry C, Xu D: IL-33 promotes ST2-dependent lung fibrosis by the induction of alternatively activated macrophages and innate lymphoid cells in mice. J Allergy Clin Immunol 2014, 134: 1422-1432.e11

37. Murthy S, Larson-Casey JL, Ryan AJ, He C, Kobzik L, Carter AB: Alternative activation of macrophages and pulmonary fibrosis are modulated by scavenger receptor, macrophage receptor with collagenous structure. FASEB J 2015, 29:3527-3536

38. Mantovani A, Biswas SK, Galdiero MR, Sica A, Locati M: Macrophage plasticity and polarization in tissue repair and remodelling. J Pathol 2013, 229:176-185

39. Bozinovski S, Anthony D, Vlahos R: Targeting pro-resolution pathways to combat chronic inflammation in COPD. J Thorac Dis 2014 6:1548-1556

40. Bozinovski S, Uddin M, Vlahos R, Thompson M, McQualter JL, Merritt AS, Wark PA, Hutchinson A, Irving LB, Levy BD, Anderson GP: Serum amyloid A opposes lipoxin A(4) to mediate glucocorticoid refractory lung inflammation in chronic obstructive pulmonary disease. Proc Natl Acad Sci U S A 2012, 109:935-940

41. Kalinski P: Regulation of immune responses by prostaglandin E2. J Immunol 2012, 188:21-28 
42. Levy BD, Clish CB, Schmidt B, Gronert K, Serhan CN: Lipid mediator class switching during acute inflammation: signals in resolution. Nat Immunol 2001, 2:612-619

43. Bozinovski S, Anthony D, Anderson GP, Irving LB, Levy BD, Vlahos R: Treating neutrophilic inflammation in COPD by targeting ALX/FPR2 resolution pathways. Pharmacol Ther 2013, 140: 280-289

44. Cooray SN, Gobbetti T, Montero-Melendez T, McArthur S, Thompson D, Clark AJ, Flower RJ, Perretti M: Ligand-specific conformational change of the G-protein-coupled receptor ALX/FPR2 determines proresolving functional responses. Proc Natl Acad Sci U S A 2013, 110:18232-18237

45. Calero C, Arellano E, Lopez-Villalobos JL, Sanchez-Lopez V, Moreno-Mata N, Lopez-Campos JL: Differential expression of Creactive protein and serum amyloid $\mathrm{A}$ in different cell types in the lung tissue of chronic obstructive pulmonary disease patients. BMC Pulm Med 2014, 14:95

46. Balode L, Strazda G, Jurka N, Kopeika U, Kislina A, Bukovskis M, Beinare M, Gardjusina V, Taivans I: Lipoxygenase-derived arachidonic acid metabolites in chronic obstructive pulmonary disease. Medicina 2012, 48:292-298

47. Takanashi S, Hasegawa Y, Kanehira Y, Yamamoto K, Fujimoto K, Satoh K, Okamura K: Interleukin-10 level in sputum is reduced in bronchial asthma, COPD and in smokers. Eur Respir J 1999, 14: 309-314

48. Recchiuti A, Krishnamoorthy S, Fredman G, Chiang N, Serhan CN: MicroRNAs in resolution of acute inflammation: identification of novel resolvin D1-miRNA circuits. FASEB J 2011, 25:544-560
49. Hsiao HM, Thatcher TH, Levy EP, Fulton RA, Owens KM, Phipps RP, Sime PJ: Resolvin D1 attenuates polyinosinicpolycytidylic acid-induced inflammatory signaling in human airway epithelial cells via TAK1. J Immunol 2014, 193:4980-4987

50. Seki H, Fukunaga K, Arita M, Arai H, Nakanishi H, Taguchi R, Miyasho T, Takamiya R, Asano K, Ishizaka A, Takeda J, Levy BD: The anti-inflammatory and proresolving mediator resolvin E1 protects mice from bacterial pneumonia and acute lung injury. J Immunol 2010, 184:836-843

51. Li L, Wu Y, Wang Y, Wu J, Song L, Xian W, Yuan S, Pei L, Shang Y: Resolvin D1 promotes the interleukin-4-induced alternative activation in BV-2 microglial cells. J Neuroinflammation 2014, 11:72

52. Spite M, Summers L, Porter TF, Srivastava S, Bhatnagar A, Serhan CN: Resolvin D1 controls inflammation initiated by glutathione-lipid conjugates formed during oxidative stress. Br J Pharmacol 2009, 158:1062-1073

53. Jung TW, Hwang HJ, Hong HC, Choi HY, Yoo HJ, Baik SH, Choi KM: Resolvin D1 reduces ER stress-induced apoptosis and triglyceride accumulation through JNK pathway in HepG2 cells. Mol Cell Endocrinol 2014, 391:30-40

54. Lee HN, Surh YJ: Resolvin D1-mediated NOX2 inactivation rescues macrophages undertaking efferocytosis from oxidative stress-induced apoptosis. Biochem Pharmacol 2013, 86:759-769

55. Schwab JM, Chiang N, Arita M, Serhan CN: Resolvin E1 and protectin D1 activate inflammation-resolution programmes. Nature 2007, 447:869-874

56. Barnes PJ: New anti-inflammatory targets for chronic obstructive pulmonary disease. Nat Rev Drug Discov 2013, 12:543-559 